\section{SAT0291 NON-REPORTING OF SYSTEMIC LUPUS ERYTHEMATOSUS IN DEATH CERTIFICATES OF LUPUS PATIENTS: ITS EXTENT AND PREDICTORS}

I. Padjen ${ }^{1,2}$, M. Erceg ${ }^{3}$, M. Cerovec ${ }^{1,2}$, M. Mayer ${ }^{1,2}$, R. Stevanović ${ }^{3}$, B. Anić ${ }^{1,2}$ ${ }^{1}$ Department of Internal Medicine, Division of Clinical Immunology and Rheumatology, University Hospital Centre Zagreb; '² University of Zagreb, School of Medicine, ${ }^{3}$ Croatian Institute of Public Health, Zagreb, Croatia

Background: Systemic lupus erythematosus (SLE) is frequently not reported in death certificates of lupus patients, despite its known role as an underlying and/or immediate cause of death. Possible reasons may be insufficient access to patients' medical records at time of death (including details on their medical history) and/or physicians' unawareness of the contribution of SLE to death.

Objectives: We aimed to analyze the extent and predictors of non-reporting of SLE in death certificates of 90 deceased SLE patients regularly followed-up in a routine academic setting at our Department.

Methods: We retrospectively observed 90 SLE patients (68 females) deceased within the 2002-2011 period. All patients were $>18$ years of age and Croatian residents at the time of death, fulfilling $\geq 4$ classification criteria of the American College of Rheumatology (ACR). We identified patients with SLE listed as a cause of death in the death certificate. An extensive set of variables was compared between patients with and without SLE reported in the certificate: demographics, ACR criteria at time of death and damage according to the Systemic Lupus Erythematosus International Collaborating Clinics (SLICC)/ACR index and its components at the time of death. We also compared the proportion of in-hospital deaths and autopsies performed. Frequencies were compared using the $x^{2}$ and Fisher's exact test, and continuous variables using the t-test and Mann-Whitney $U$ test. Variables associated with reporting of SLE in the death certificate in the univariate analysis were included in a multivariate logistic regression model.

Results: SLE was reported in death certificates of $41 / 90(46 \%)$ patients. Patients with SLE not reported in their death certificates were older at death $(62 \pm 14$ vs. $53 \pm 15$ years) and diagnosis ( $53 \pm 14$ vs $42 \pm 18$ years) and had a longer time from their last visit at our Department to death $(0.80 \pm 1.00$ vs. $0.34 \pm 0.66$ years), compared to patients with SLE listed in the death certificate $(p<0.05)$. They also had a lower proportion of renal disorder (20/49 vs. 29/41), cardiovascular and pulmonary damage (18/49 vs. $28 / 41$ and $7 / 49$ vs. $13 / 41$, respectively), and died less frequently in hospital (28/49 vs. $35 / 41)$ and due to infections (4/49 vs. $26 / 41)$ $(p<0.05)$. Conversely, these patients had a higher frequency of malignancy as a feature of damage (17/49 vs. 6/41) and a cause of death (14/49 vs. 1/41) $(p<0.05)$. Only patients without SLE listed in their death certificate accrued gastrointestinal damage ( $7 / 49$ vs. $0 / 41, p=0.015)$, hence this type of damage could not be included in the multivariate logistic model. In the multivariate model, the presence of infection as a cause of death was the single variable related to (non-)reporting of SLE (OR 0.053; 95\% Cl 0.012-0.237) (Table 1).

Table 1. Predictors of non-reporting of SLE in death certificates (OR: odds ratio; $\mathrm{Cl}$ : confidence interval)

\begin{tabular}{lccc} 
Variable & OR & $\mathbf{9 5 \%} \mathbf{C l}$ & $\mathbf{p}$ \\
\hline Age at diagnosis (years) & 1.050 & $0.961-1.148$ & 0.280 \\
Age at death (years) & 0.996 & $0.906-1.096$ & 0.938 \\
Time from last visit to death (years) & 1.122 & $0.496-2.542$ & 0.782 \\
Renal disorder (yes/no) & 0.434 & $0.126-1.499$ & 0.187 \\
Pulmonary damage (yes/no) & 0.536 & $0.119-2.406$ & 0.416 \\
Cardiovascular damage (yes/no) & 0.412 & $0.102-1.666$ & 0.214 \\
Malignancy (feature of damage) (yes/no) & 2.934 & $0.597-14.423$ & 0.185 \\
Infection as cause of death (yes/no) & 0.053 & $0.012-0.237$ & $<0.001$ \\
In-hospital death (yes/no) & 1.012 & $0.223-4.592$ & 0.987
\end{tabular}

Conclusions: Non-reporting of SLE in death certificates of lupus patients may be an obstacle towards assessing the true extent of SLE-related mortality, calling into question the reliability of vital statistics data extracted only from death certificates. Infections as causes of death and gastrointestinal damage may influence reporting of SLE in death certificates.

References:

[1] Calvo-Alen J et al. Rheumatology 2005;44:1186-9.

Disclosure of Interest: None declared

DOI: 10.1136/annrheumdis-2017-eular.2825

\section{SAT0292 LONG-TERM PROGNOSIS AND PREDICTING FACTORS OF CHINESE PATIENTS WITH ANTIPHOSPHOLIPID SYNDROME}

J. Zhao, Y. Sun, P. Zhang, Q. Wang, M. Li, X. Zeng. Peking Union Medical College Hospital, Beijing, China

Background: Antiphospholipid syndrome (APS) is an acquired autoimmune prothrombotic condition characterized by persistent circulating antiphospholipid antibodies (APL). The pathogenic mechanisms that lead to clinical manifestations associated with APL are only partially understood. And to date, long-term anticoagulation has been the only treatment shown to reduce vascular complications. Objectives: The aims of the present study were to assess and identify the prognostic factors of the long-term outcomes and mortality of antiphospholipid syndrome (APS) in Chinese patients.

Methods: Records of 160 patients with APS admitted to Peking Union Medical College Hospital in Beijing between 2005 and 2015 were investigated. Demographic characteristics, cumulative clinical and laboratory features, autoantibody profiles were retrieved from the database. Survival rates were studied by KaplanMeier method, and COX proportional hazard model was adopted to perform the analysis of predicting factors for mortality.

Results: The entire cohort consisted of 110 (68.8\%) female and $50(31.3 \%)$ male patients. Mean (SD) age at study entry was $36.5 \pm 14.9$ years. The most prevalent thrombotic risk factors were hypertension, dyslipidemia, and smoking, present in $5-15 \%$ of the total cohort. In total, $50.6 \%$ of the patients had primary APS, $45.9 \%$ had APS associated with SLE, $2.0 \%$ APS associated with other connective tissue diseases. The most prevalent immunological features at baseline were LA (71.3\%), aCL (55.0\%), and $\beta 2 \mathrm{GPI}(49.4 \%)$. No significant statistical differences were found in the clinical presentation of the APS according to the presence or absence of any of these antibodies. During the 10-year period, $16(10.0 \%)$ patients ( 8 female and 8 male) died. The overall 1, 3, and 5 -year survival rate was $92.6 \%, 89.1 \%$ and $87.1 \%$, respectively. The most common causes of death were severe thrombotic events, including pulmonary embolism, strokes and myocardial infarction $(43.8 \%$ of total deaths), infections (18.8\%). COX proportional hazard model show thrombocytopenia is the independent prognostic factor of mortality (HR 8.228, 95\% Cl 1.866-36.282).

Table 1. Baseline characteristics of APS patients

\begin{tabular}{lccc}
\hline Clinical characteristics & Prevalence & \multicolumn{2}{c}{ Thrombotic event } \\
\cline { 3 - 4 } & & No $(\mathrm{N}=51)$ & Yes $(\mathrm{N}=109)$ \\
\hline Female, n/\% & $110(68.8 \%)$ & $42(82.4 \%)$ & $68(62.4 \%)$ \\
Age, year, mean+SD & $36.5 \pm 14.9$ & $34.2 \pm 14.9$ & $37.4 \pm 14.9$ \\
Thrombotic events & & & \\
$\quad$ Arterial thrombosis & $59(36.9 \%)$ & - & $59(54.1 \%)$ \\
$\quad$ Venous thrombosis & $72(45.0 \%)$ & - & $72(66.1 \%)$ \\
$\quad$ Coexist of arterial and venous thrombosis & $22(13.8)$ & - & $22(20.2 \%)$ \\
Systemic autoimmune diseases & $79(49.4 \%)$ & $33(64.7 \%)$ & $46(42.2 \%)$ \\
Thrombophylic risk factors & & & \\
$\quad$ Smoking & $8(5.0 \%)$ & $2(3.9 \%)$ & $5(5.5 \%)$ \\
$\quad$ Dyslipidemia & $20(12.5 \%)$ & $6(11.8 \%)$ & $14(12.8 \%)$ \\
$\quad$ HTN (systolic $>140)$ & $24(15.0 \%)$ & $7(13.7 \%)$ & $17(15.6 \%)$ \\
ACL & $88(55.0 \%)$ & $32(62.7 \%)$ & $56(51.4 \%)$ \\
$\beta 2$ GP1 & $79(49.4 \%)$ & $31(60.8 \%)$ & $48(44.0 \%)$ \\
Lupus anticoagulants & $114(71.3 \%)$ & $39(76.5 \%)$ & $75(68.8 \%)$ \\
Tri-positive & $41(25.6 \%)$ & $21(41.2 \%)$ & $20(18.3 \%)$ \\
Thrombocytopenia & $71(44.4 \%)$ & $23(45.1 \%)$ & $48(44.0 \%)$ \\
Hypocomplementaemia & $59(36.9 \%)$ & $25(49.0 \%)$ & $34(31.2 \%)$ \\
\hline
\end{tabular}

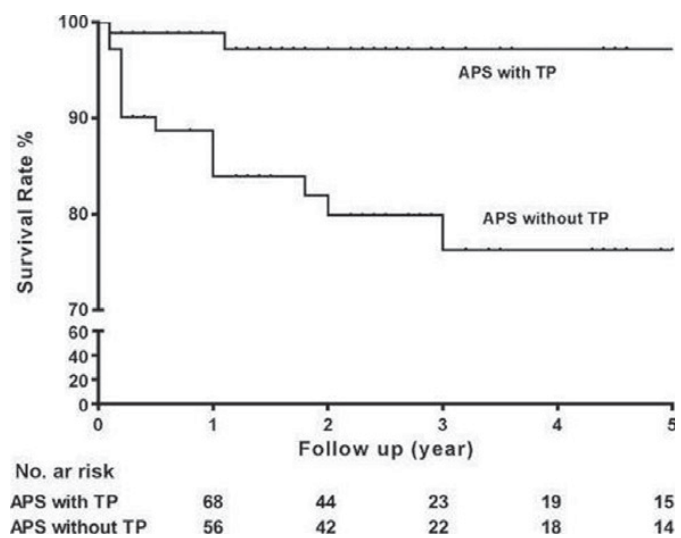

Conclusions: patients with APS develop significant morbidity and mortality despite current treatment. More attention should be devoted to APS patients with thrombocytopenia.

Disclosure of Interest: None declared

DOI: 10.1136/annrheumdis-2017-eular.5580

\section{SAT0293 PNEUMOCOCCAL INFECTION IN PATIENTS WITH SYSTEMIC LUPUS ERYTHEMATOSUS}

J. Schurder, T. Goulenok, R. Jouenne, A. Dossier, D. van Gysel, T. Papo, K. Sacre. Paris-7 University, APHP, Bichat Hospital, Paris, France

Background: A 5-fold increase in the risk of death is due to infection in systemic lupus erythematosus (SLE) patients when compared to age- and sex-matched controls Pneumococcal infection $(\mathrm{PI})$ has been reported to be more frequent and severe in SLE

Objectives: Our study aimed to analyze the risk factors associated with the occurrence and severity of $\mathrm{PI}$ in SLE patients

Methods: Medical records of all SLE patients and all patients admitted with $\mathrm{PI}$ in the Department of Internal Medicine (Bichat Hospital, Paris, France) from January 2005 to December 2014 were retrospectively reviewed. Clinical characteristics associated with PI occurrence and severity were analyzed, both in SLE and non SLE patients.

Results: One hundred and ninety SLE patients ( $42.2+14.9$ years; $87.4 \%$ females) were hospitalized over a 10-year period. PI was the reason for admission in 6 (3.2\%) patients, including 5 cases of invasive infection. With a follow-up of 2112.8 
years for the total cohort, incidence of invasive PI in SLE was of 236/100.000 patient-years. As compared to the incidence in general French population, invasive $\mathrm{PI}$ was 26 times more frequent in SLE patients. PI occurred at a younger age $(43.5+14.9$ versus $65.3+18.7$ years, $p=0.009)$ and was more severe, with a higher frequency of invasive infection $(p<0.001)$ and higher need for ICU admission $(p=0.015)$ in SLE as compared to non SLE patients. Of note, unusual PI sites, including pneumococcal endocarditis $(n=1)$, arthritis $(n=1)$ and peritonitis $(n=1)$ were observed in SLE patients only. Risk factors associated with PI in SLE patients were a serum gammaglobulin level $<5 \mathrm{~g} / \mathrm{L}(\mathrm{p}=0.003)$ and a past history of lupus nephritis $(p=0.047)$, only. Steroids $(p<0.001)$ and immunosuppressive drugs $(p=0.027)$ were associated with infection severity.

Conclusions: Pneumococcal infections occur at a younger age, are more frequent and severe in SLE patients. Hypogammaglobulinemia and lupus nephritis increased the risk for $\mathrm{PI}$, whereas steroids and immunosuppressive drugs were associated with infection severity only. Our study shows that SLE patients have an increased risk for invasive $\mathrm{PI}$ and points to the need for vaccination against streptococcus pneumoniae in SLE.

Disclosure of Interest: None declared

DOI: 10.1136/annrheumdis-2017-eular.1016

\section{SAT0294 COMPARISON OF CLINICAL CARE BETWEEN CHINESE AND AMERICAN PATIENTS WITH SYSTEMIC LUPUS ERYTHEMATOSUS}

J. Dong ${ }^{1}$, H. Ma ${ }^{1}$, W.N. Roberts ${ }^{2}$, L. Wang ${ }^{1}$, F. Khan ${ }^{3}$, K.A. Lyn Shue ${ }^{2}$, L. Zhao ${ }^{1}$, L. Pan ${ }^{1} .{ }^{1}$ Rheumatology and Clinical Immunology, Affiliated Hospital of Qingdao University, Qingdao, China; ${ }^{2}$ Rheumatology; ${ }^{3}$ Internal Medicine, University of Louisville, Louisville, KY, United States

Background: In addition to gender and ethnicity, modifiable variables like geography, socioeconomic status, health system structure, education, and physician expertise may influence outcomes in systemic lupus erythematosus (SLE).

Objectives: To compare characteristics of and treatment options for subsets of Chinese and American patients with SLE to elucidate factors that contribute to disease activity and damage.

Methods: Chart review of 77 Chinese (Qingdao) and 48 Midwestern American (Louisville, Kentucky) patients meeting American College of Rheumatology (ACR) criteria for a diagnosis of SLE followed up for four years were analyzed retrospectively. Organ damage was assessed using the Systemic Lupus International Collaborating Clinics (SLICC)/ACR Damage Index (SDI), and disease activity was assessed using the Systemic Lupus Erythematosus Disease Activity Index (SLEDAI). Statistics were parametric exploratory tests of significance and multiple regression analyses in this hypothesis-generating effort.

Results: The interval between the time of onset and diagnosis was 44 months shorter in the Chinese arm $(p=0.001)$, and Chinese patients followed up at six times greater frequency than American patients $(p<0.001)$. Despite the lack of formal matching, the two cohorts featured similar disease activity according to the SLEDAI. Based on the SDI, rates of organ damage were higher in the American group. Chinese patients received more steroids, cyclophosphamide, hydroxychloroquine, intravenous immune globulin, and cyclosporine than the Louisville group, while the Louisville patients received more mycophenolate mofetil and azathioprine $(p=0.001)$.

\begin{tabular}{lccc} 
Table 1 & & \\
\hline Variable & $\begin{array}{c}\text { Qingdao }(n=77), \\
\text { mean } \pm \text { SD }\end{array}$ & $\begin{array}{c}\text { Louisville }(n=48), \\
\text { mean } \pm \text { SD }\end{array}$ & $p$-value \\
\hline Onset age (years) & $30.24 \pm 11.95$ & $30.21 \pm 12.21$ & 0.989 \\
Age at diagnosis (years) & $30.89 \pm 11.92$ & $34.5 \pm 12.99$ & 0.114 \\
$\begin{array}{l}\text { Duration between SLE onset and } \\
\quad \text { diagnosis (months) }\end{array}$ & $7.94 \pm 18.46$ & $52.30 \pm 89.90$ & 0.001 \\
Clinic visits per year & $10.93 \pm 7.09$ & $3.02 \pm 1.91$ & $<0.001$ \\
$\quad$ Interval between the last two times of & & & \\
$\quad$ follow up (months) & $1.89 \pm 1.31$ & $12.32 \pm 28.32$ & 0.014 \\
Disease duration (years) & $5.97 \pm 5.72$ & $5.22 \pm 5.53$ & 0.466 \\
SLEDAl & $5.81 \pm 4.32$ & $4.63 \pm 4.77$ & 0.156 \\
SDI & $0.44 \pm 0.64$ & $1.23 \pm 1.057$ & $<0.001$ \\
\hline
\end{tabular}

Table 2

\begin{tabular}{lccc}
\hline Medication & Qingdao $(n=77)$ & Louisville $(n=48)$ & $p$-value \\
\hline Prednisone & $77(100 \%)$ & $29(60.42 \%)$ & $<0.0001$ \\
Cyclophosphamide & $34(44.16 \%)$ & $6(12.50 \%)$ & $<0.0001$ \\
Hydroxychloroquine & $72(93.51 \%)$ & $32(66.67 \%)$ & $<0.0001$ \\
Methotrexate & $9(11.69 \%)$ & $3(6.25 \%)$ & 0.489 \\
Mycophenolate mofetil & $10(12.99 \%)$ & $18(37.50 \%)$ & 0.001 \\
Azathioprine & $2(2.60 \%)$ & $5(10.42 \%)$ & 0.147 \\
Intravenous immune globulin & $12(15.58 \%)$ & $1(2.08 \%)$ & 0.035 \\
Cyclosporine & $17(22.08 \%)$ & $1(2.08 \%)$ & 0.005 \\
\hline
\end{tabular}

Conclusions: The establishment of follow-up and treatment of SLE differs in specific, identifiable ways between these subsets of Chinese and midwestern American patients. Greater access to and increased frequency of follow-up appears associated with a lesser degree of organ damage, supporting the treat-to-target concept as applied to SLE. Complete, controlled trials in both settings are necessary, and further detailed comparison of larger cohorts may inform conclusions about the likelihood of generalizability of trial results from one setting to another.

Disclosure of Interest: None declared

DOI: 10.1136/annrheumdis-2017-eular.5561

\section{SAT0295 ANTI-RO52/TRIM21 ANTIBODIES ARE ASSOCIATED WITH QT INTERVAL PROLONGATION IN PATIENTS WITH SYSTEMIC LUPUS ERYTHEMATOSUS}

L.F. Perez, L.H. Silveira, L. Amezcua, O. Estevez, M. Moreno. Reumatologia, Instituto Nacional de Cardiologia Ignacio Chavez, Mexico City, Mexico

Background: Long QT syndrome (LQTS) is characterized by an abnormal QT corrected (QTC) interval prolongation that is associated with increased risk of sudden death. Studies have associated LQTS with several rheumatic conditions, and evidence points towards a link between the degree of systemic inflammation and the duration of QTc interval. Moreover, recent evidence suggests that anti-Ro antibodies may play a role in the QTc prolongation by mechanisms not fully understood, thus constituting a novel autoimmune-mediated LQTS.

Objectives: This study was aimed to assess whether QTc interval prolongation is associated with the presence of anti-Ro antibodies in SLE, particularly with reactivities against Ro52/TRIM21 antigens.

Methods: Consecutive patients fulfilling the 1997 ACR criteria for SLE were included. Patients with history of ischemic heart disease, with implantable pacemakers, and those taking drugs that potentially could affect QT interval (except for antimalarials) were excluded. Patients underwent a resting 12-lead electrocardiogram recording to measure QT interval corrected by BazzetÕs formula. A QTc interval duration greater than $460 \mathrm{msec}$ in women and $440 \mathrm{msec}$ in men was set to be abnormal. Serum anti-Ro and anti-Ro52/TRIM21 antibody levels were measured by ELISA. Data were expressed as frequencies and means ( \pm standard deviation), and differences were tested by YatesÕ continuity corrected chi square or Mann-Whitney tests, while linear regressions were performed to assess linearity between autoantibody levels and QTc duration. The GraphPad Prism 4.02 software was used for calculations.

Results: Sixty-six patients with mean age of $39 \pm 13$ years (57 female gender) were included. A QTc prolongation was found in 10 patients $(15 \%)$, with mean QTc interval of $470 \pm 18 \mathrm{msec}$ as compared to $414 \pm 23 \mathrm{msec}$ in those with no LQTS. Main clinical and demographic characteristics were similar for both groups, except for a lesser use of antimalarials and higher serum creatinine levels in patients with LQTS. Disease activity was similar between groups.Anti-Ro antibody levels were significantly higher in patients with prolonged QT interval ( $75 \pm 66 \mathrm{U} / \mathrm{mL}$ versus $29 \pm 44 \mathrm{U} / \mathrm{mL} ; \mathrm{P}=0.005)$; similarly, anti-Ro52/TRIM21 levels were higher in those with LQTS $(50 \pm 55 \mathrm{U} / \mathrm{mL}$ versus $14 \pm 30 \mathrm{U} / \mathrm{mL} ; P=0.01)$. Notably, a linear association (see the Figure) between the QTc intervals and levels of anti-Ro antibodies ( $\mathrm{r} 2=0.073 ; \mathrm{P}=0.02)$ and anti-Ro52/TRIM21antibodies $(\mathrm{r} 2=0.078 ; \mathrm{P}=0.02)$ was observed.
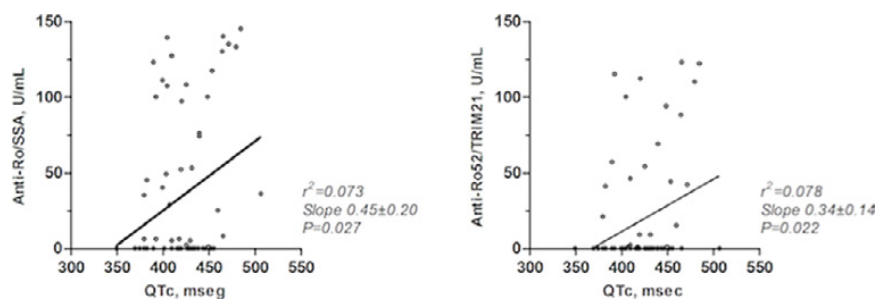

Conclusions: Our results strengthen the hypothesis that a specific autoantibodymediated LQTS occur in SLE patients positive to anti-Ro antibodies. This interference in the ventricular repolarization appears to be associated with increased levels of antibodies against Ro52/TRIM21 antigens, and supports the realization of an electrocardiogram as part of the routinely evaluation in SLE patient with circulating anti-Ro antibodies.

Disclosure of Interest: None declared

DOI: 10.1136/annrheumdis-2017-eular.6695

\section{SAT0296 RELATIONSHIP BETWEEN DISEASE ACTIVITY INDEX SCORES AND SUBJECTIVE ASSESSMENTS IN EARLY SYSTEMIC LUPUS ERYTHEMATOSUS}

M. Garabajiu ${ }^{1}$, V. Sadovici-Bobeica ${ }^{1}$, L. Mazur-Nicorici ${ }^{1}$, M. Cebanu ${ }^{1}$, V. Salaru ${ }^{2}$, M. Mazur ${ }^{1} .{ }^{1}$ Rheumatology; ${ }^{2}$ Family Medicine, State University of Medicine and Pharmacy "Nicolae Testemitanu", Chisinau, Moldova, Republic of

Objectives: To evaluate the disease activity in patients with early systemic lupus erythematosus (early SLE) and to compare it to patient's and physician's global assessment.

Methods: Cross-sectional study including 41 early SLE patients that fulfilled SLICC classification criteria, 2012. The early disease was defined one with the duration 2 years from the diagnosis. The disease activity was assessed by SLEDAI-2K and SLAM. Global indices were appreciated by patient and physician global assessments (PGA and MDGA), rated by $0-100$ numeric score. We correlated disease activity indices with global assessments by Pearson coefficient. 